\section{Appleton Tower}

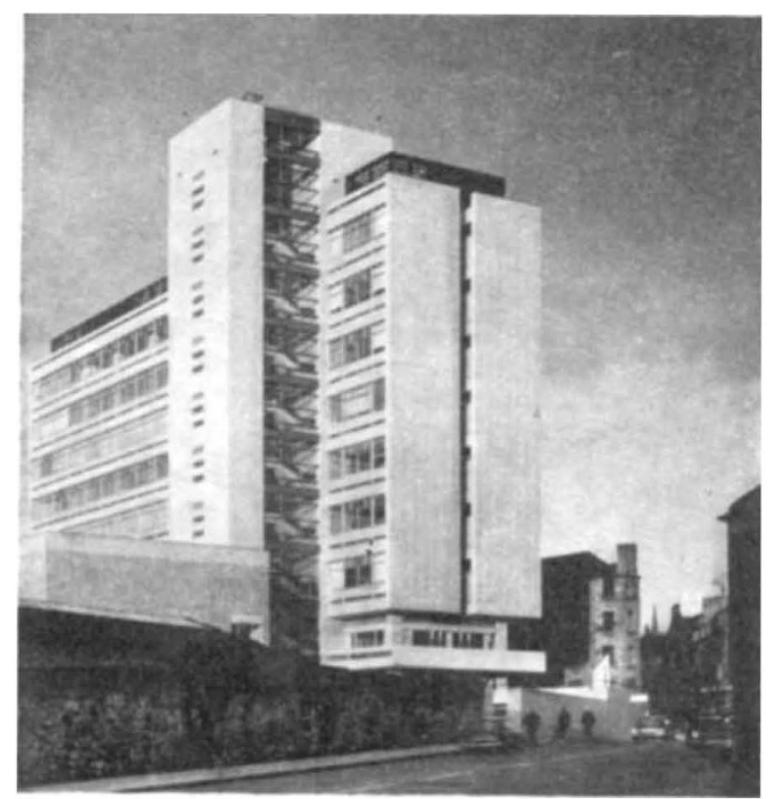

THE east gable of the recently inaugurated Appleton Tower of the University of Edinburgh. The building was designed by Mr. Alan Reiach for the use of first year science students and named after the late Sir Edward Appleton (Principal and Vice-Chancellor of the University until his death in 1965). It consists of seven floors of teaching laboratories with ancillary facilities and a block with five lecture theatres able to seat 1,250 altogether. The tower post-dates its companion tower, the Hume Tower (not shown), by some three years and like it (writes an architectural correspondent) offers the compensation of excellence in its effect on its immediate environment, in its handling of function and space and in its quality of detail and finish. Away from central Edinburgh, city of fragile pinnacles, they would be beyond reproach. But in the eyes of many citizens, their university has dealt the cherished skyline a second knock. To them the new tower does not convey a message -of cool and slender elegance. In all fairness, however, it must be admitted that the economics of tower building are such that bulkiness is ever a hazard to success and the architectural problem posed to the designer of both these buildings was nothing if not severe. While the suavity of the design of the towers has gone far to mitigate the adverse effects of such large buildings, an architectural language is still required which can be equally understood by client, architect, planner and public at large.

\section{Ribosome Proteins}

\section{from a Correspondent in Molecular Biology}

Although the way in which ribosomes function in the promotion of the synthesis of polypeptide chains is obscure, it is perhaps hardest of all to explain the reasons for the remarkable multiplicity of their structural proteins. These are nearly all basic, have relatively low molecular weights and (at least in Escherichia coli) show essentially no other $\mathrm{N}$-terminal amino-acids than alanine and methionine. An almost incontestable body of evidence appears by now to have built up that these components are genuine, and not artefacts of the preparation.

This year's Abstract issue of Federation Proceedings has now appeared, and among the 3,500 abstracts a few nuggets of gold as usual lie buried. In particular there are a number of interesting reports bearing on the nature and function of the ribosomal proteins. It is now well known that by centrifugation in concentrated caesium chloride solutions a sizable fraction of the proteins may be removed from $E$. coli ribosomes: the $50 \mathrm{~S}$ and $30 \mathrm{~S}$ ribosomal sub-units give place to protein-deficient "cores" of $40 \mathrm{~S}$ and $23 \mathrm{~S}$. Traub and Nomura (Fed. Proc., 26, 285; 1967) have now taken the fractionation further, and demonstrated the existence of functionally distinct sets of ribosomal proteins. Thus the proteins which are extracted in caesium chloride are electrophoretically distinguishable from those remaining in the cores of both sub-units. The cores are inert, and will not incorporate aminoacids or bind transfer RNA, but fully functional $50 S$ and $30 S$ sub-units are regenerated when the cores are mixed with the corresponding separated proteins. Traub and Nomura have now fractionated the separated proteins from both sub-units into two groups by ion-exchange chromatography. These were then returned separately to the cores, and the following gratifying results are reported: only one of the two groups of the separated proteins from the $50 S$ particles proved essential for protein synthesis, the others having some kind of enhancing effect; similarly, only the one set of proteins was required by the smaller sub-unit for amino-acid incorporation, as well as specific binding of transfer RNA, and again the remaining proteins enhanced both functions.

The relation of these results to another report in the same issue (Gottesman, ibid., 612) is not yet clear. Here the ribosomes are washed with $0.5 \mathrm{M}$ ammonium chloride, and protein-depleted particles are said to result. Presumably the extent of depletion is less than in the caesium chloride-treated ribosomes, for some amino-acid incorporation can be made to occur. It is found, in fact, that only one peptide bond is created in the poly A-lysine system, but that when the ammonium chloride supernatant and GTP are added, polypeptide synthesis proceeds normally. The putative peptide synthetase thus appears to be present in the depleted ribosomes. Analysis of the stripped proteins is not reported. Further fractionation of the ribosomal proteins will doubtless produce some interesting results. It may be mentioned that core particles have now been prepared also from mammalian ribosomes (Petermann et al., ibid., 285), which appear not to be susceptible to caesium chloride, by a procedure involving the use of lithium chloride.

Another approach to ribosome function comes from Szer and Nowak (J. Mol. Biol., 24, 333; 1967), who report the interesting observation that polydihydrouridylic and poly N-methyluridylic acids, neither of which is capable of forming a two-stranded complex with poly A, though they have no messenger activity, attach to the messenger binding site (in competition with poly $\mathrm{U}$ ) of the $30 \mathrm{~S}$ sub-unit. This strongly sup. ports earlier evidence that the messenger is not held by base-pairing, but rather by some interaction most probably involving the messenger phosphate groups, and perhaps ribosomal proteins. 